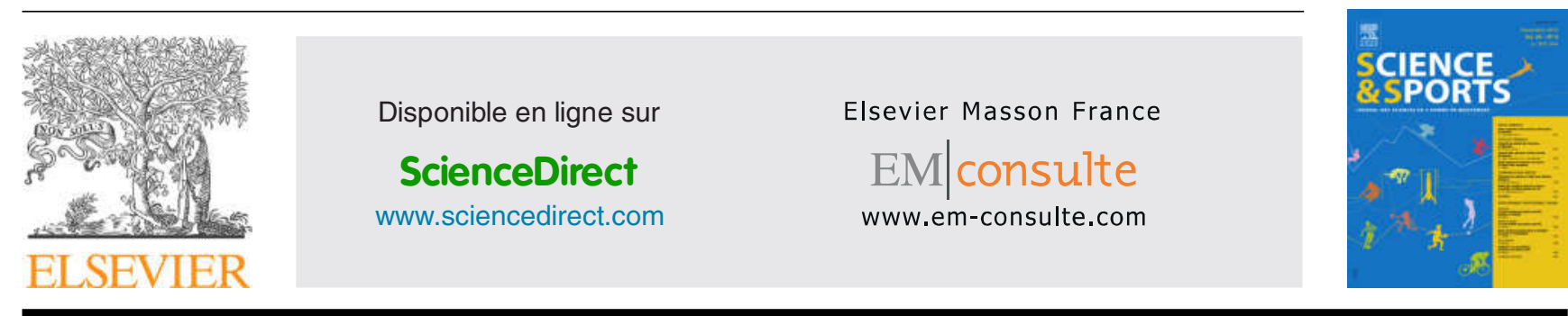

ORIGINAL ARTICLE

\title{
Effects of transcranial direct current stimulation on joint flexibility and pain in sedentary male individuals
}

\section{Effets de la stimulation directe sur la des articulations et la douleur chez des personnes masculines}

Q2 V. Lins ${ }^{a, *}$, E. Lattari ${ }^{a}$, D. Monteiro
G.c Albuquerque Neto $^{d}$, S. Machado ${ }^{\text {a,e }}$

\footnotetext{
a Physical Activity Neuroscience Laboratory, Physical Activity Sciences Post-Graduate Program (PGCAF), Salgado de Oliveira University (UNIVERSO), RJ, Brazil

b Sport Science School of Rio Maior-Polytechnic Institute of Portugal

c Research Center in Sport, Health and Human Development (CIDESD), Portugal

d Physical Activity Sciences Post-Graduate Program (PGCAF), Salgado de Oliveira University (UNIVERSO), RJ, Brazil

Q3 e Intercontinental Neuroscience Research Group
}

Received 3 April 2018; accepted 25 January 2019

\section{KEYWORDS}

Non-invasive brain stimulation;

Transcranial direct current stimulation;

Flexibility;

Pain;

Primary motor cortex

\begin{abstract}
Summary The aim of this study was to analyze the effects of cathodal tDCS (c-tDCS) on joint flexibility and pain perception in a sedentary male. Eight male healthy, sedentary rightleg-dominant and novice in muscle stretching aged between 19 and 30 years $(24.0 \pm 4.0$ years) were recruited. Subjects performed three experimental conditions in a randomized, double-blinded crossover design: anodal stimulation (a-tDCS), c-tDCS and sham-tDCS (2 mA for 20 minutes targeting the bilaterally motor cortex). Before and immediately after the experimental conditions (baseline and post-condition, respectively), subjects completed the range of motion (ROM) of right hip test and the Visual Analogic Scale for Pain (VAS pain; level of significance $P<0.05)$. In post-condition, c-tDCS was greater than to a-tDCS $(P<0.001)$, and sham-tDCS $(P<0.001)$ in the right hip ROM. Hip ROM increased in the post-condition compared to baseline in the $\mathrm{c}$-tDCS condition $(P<0.001)$. In the a-tDCS condition, hip ROM decreased in the post-condition compared to baseline $(P<0.001)$. For VAS pain, in post-condition,
\end{abstract}

\footnotetext{
* Corresponding author. Laboratory of Physical Activity Neuroscience, Physical Activity Sciences Post-graduate Program, Salgado de Oliveira University, 22061-021 Niterói, Rio de Janeiro, Brazil.

E-mail address: secm80@gmail.com (V. Lins).
} 


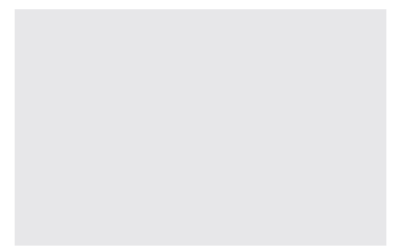

\section{MOTS}

Stimulation cérébrale non invasive ; Stimulation

courant continu ;

Flexibilité ;

Douleur ;

Cortex moteur

primaire

Stretching refers to a movement applied to increase the range of motion (ROM) of joints, i.e. flexibility, and have been used by sports coaches for performance enhancement and injury prevention by regaining joint ROM [1]. Traditionally, stretching exercises have been studied in relation to injury [2], performance [3], and muscle soreness [4]. The stretching is an effective method to chronically increase the joint range of motion [5]. This increase in ROM has been related to both neural $[6,7]$ and mechanical [8] factors. More recently, a sensory theory has been proposed suggesting instead that increases in muscle extensibility are due to a modification of sensation only [9].

Different forms of stretching exercises are an effective way of improving ROM in healthy individuals $[10,11]$ and other techniques have been proposed for improvement of the ROM as the yoga [12] and transcranial direct current stimulation [13]. Transcranial direct current stimulation (tDCS) consists of a non-invasive electrical stimulus that can promote excitation, through tonic depolarization of the membrane resting potential (anodal stimulus, a-tDCS), or cortical inhibition, by hyperpolarization of the membrane resting potential (cathodal stimulus, c-tDCS) $[14,15]$. This non-invasive neuromodulatory technique has been used as an ergogenic in healthy subjects to induce changes in physical performance, such as increase in muscular strength
$[16,17]$, and joint flexibility [18], and reduction in ratings perceived exertion [16,18], and pain [19].

Only one study investigated the effects of tDCS on joint flexibility in healthy subjects [13]. In this study, c-tDCS over the sensorimotor cortex (SM1), with a current of 10 minutes and intensity of $2.0 \mathrm{~mA}$, resulted in a $10.5 \%$ increase in ROM of the ankle in eight male healthy [13]. The author's suggestion that the SM1 was involved in joint flexibility because the passive torque did not change and this may have been affected neural factors, such as perception of joint angle or pain. Corroborating this hypothesis, the c-tDCS applied over cortical regions of the pain neuromatrix, as the SM1, increased pain thresholds in healthy adults [20]. To test the hypothesis that the SM1 is involved in joint flexibility, we investigated whether c-tDCS over SM1 bilaterally modifies hip ROM and decrease pain perception in the sedentary male. Thus, the aim of this study was to investigate whether the effects of c-tDCS on joint flexibility and pain in sedentary male, would enhance hip ROM, and decrease pain perception in comparison to a-tDCS and sham-tDCS.

\section{Methods}

\subsection{Subjects}

Eight male healthy, sedentary [21] and novice in muscle stretching, right-leg-dominant, and aged between 19 
and 30 years $(24.0 \pm 4.0$ years $)$ were recruited. Regarding anthropometric measurements, participants averaged $84.1 \pm 17.6 \mathrm{~kg}$ of body mass and $173.6 \pm 4.1 \mathrm{~cm}$ of height. Were excluded subjects that had neuropsychiatric, cardiovascular, or osteoarticular diseases, used any kind of neuropsychiatric drugs, and used any caffeinated beverage on the day of the experiment or alcoholic beverages in the day before. The sample size was calculated using G*Power software (version 3.1). For analysis we use the following commands: Test family $=$ F-tests, Statistical test $=$ analysis of variance (Anova): repeated measures between factors, $\alpha$ error probability $=0.05$, power $(1-\beta$ error probability $)=0.80$, and effect size was set with $d=0.54$ [13]. This effect size was calculated according to the mean and standard deviation data of c-tDCS and sham-tDCS interventions [13]. A total of 5 participants in each condition were needed for this study. Each participant signed a written consent form, and the experiment was approved by the institutional ethics committee of the Salgado Oliveira University, according to the Norms of Conduct in Human Research (CNS resolution 466/2012).

\subsection{Anthropometric measurements}

Participants' body mass and height were measured with a weighing scale and stadiometer (Filizola model 31; Filizola S.A., Paulo, Brazil), following the recommendations proposed by the International Society for Advancement of Kinanthropometry [21].

\subsection{Application of transcranial direct current stimulation (tDCS)}

The subjects remained seated comfortably in a chair located within the laboratory. The electric current of $2 \mathrm{~mA}$ was applied using a pair of pads soaked in saline solution $(\mathrm{NaCl}$ $140 \mathrm{mmol}$ dissolved in Milli-Q water) comprising the two $5 \times 5 \mathrm{~cm}$ electrodes, connected to a direct current stimulation device (TCT, China) and positioned using elastics. The stimulation procedures had the duration of 20 minutes. The procedures for placing the electrodes followed the proposals of Mizuno et al. [13]. For c-tDSC, the cathode electrode is placed over the SM1 bilaterally and anode electrode was placed over the occipital cortex $(\mathrm{OC})$, both located on electrode area $\mathrm{Cz}$ and $\mathrm{Oz}$, in accordance with the international 10-20 system EEG [22]. For a-tDCS the anode was placed over the SM1 and cathode was placed over the OC. In the sham-tDCS condition, the electrodes were placed in the same positions of the a-tDCS. However, the stimulator was turned off after 30 seconds, acting as a placebo condition [23]. Patients usually report tingling sensations or itching from the initial electrical stimulation but there is evidence that there are no stimulation effects has the device is turned off during the remaining time. This procedure allows the subjects to become blinded to the type of stimulus that they will receive during the experiment [24]. All tDCS procedures were conducted by the same research assistant.

\subsection{ROM of the hip (hip ROM)}

To evaluate the range of motion of the joint hip, an angular test was used, as proposed by the American College of Sports Medicine (ACSM) [21], with the use of a digital goniometer (IGAGING ${ }^{\circledR}$, Paulo, Brazil). The subject was positioned in dorsal decubitus, with the hip at zero degrees of flexion, extension, adduction, abduction, and rotation. A first evaluator kept the subject's contralateral leg fixed to the stretcher while passively raised the other to the highest level of discomfort or pain reported by the subject, keeping the knee extended, and the foot in a neutral position by a single attempt. Then, the second evaluator performed the measurement of the angle reached by the maximum ROM in the joint right hip. The goniometer was positioned as follows: Fulcrus = major trochanter of the femur; Stabilization branch = lateral midline of the pelvis; Mobile branch = lateral midline of the femur, using the lateral epicondyle as the reference point.

\subsection{Visual Analogic Scale for Pain (VAS pain)}

The VAS pain is a unidimensional measure of pain intensity valid, reliable and appropriate for use in clinical practice [25]. For pain intensity, the scale is most commonly anchored by "no pain" (score of 0 ) and "pain as bad as it could be" or "worst imaginable pain"' (score of 10 [100-mm scale]) [26].

\subsection{Experimental procedures}

Each participant had 4 visits to the laboratory. On the first visit, subjects assigned the consent form, completed a socio-demographic questionnaire, and were submitted to anthropometric measurement. The subjects had to perform a warm-up on the cycle ergometer, with duration of 5 minutes, $70 \mathrm{rpm}$ and the initial load $(\mathrm{kg})$ was adjusted according to body weight before performing the ROM of the right hip test. After warm-up, the subjects performed the ROM of the right hip test and during the maximum Hip ROM, the pain scale was measured through the VAS Pain [26]. The procedures of the first visit were used as familiarization to the later experimental procedures. Following the initial visit, with 48 to 72 hours of the intervals between the visits, subjects attended the lab for the three experimental conditions (c-tDCS, a-tDCS, or sham-tDCS), with session order randomly counterbalanced across participants. The randomization scheme was generated by using the Web site Randomization.com (http://www.randomization.com). Before and after the experimental conditions (baseline, and post-stimulation), subjects performed the warm-up, ROM of the hip (hip ROM) and the VAS pain during test performance (Fig. 1). All sessions were performed in the afternoon (i.e., 14:00-17:00-hour p.m.) to avoid circadian effects on the flexibility. The ambient temperature ranged from $21^{\circ} \mathrm{C}$ to $23^{\circ} \mathrm{C}$ and relative humidity ranged from 55 to $70 \%$. Subjects were also informed to maintain their regular food and hydration diet before performing the visits and were discouraged to consume ergogenic beverages like coffee. The Hip ROM and VAS Pain were conducted by two researchers and tDCS was conducted for another research assistant. 


\section{Experimental conditions}

\section{Baseline \\ Baseline}

\begin{tabular}{|c|}
\hline Warm-up \\
$\downarrow$ \\
Hip ROM \\
$\downarrow$ \\
VAS Pain \\
\hline
\end{tabular}

\section{Post-condition}

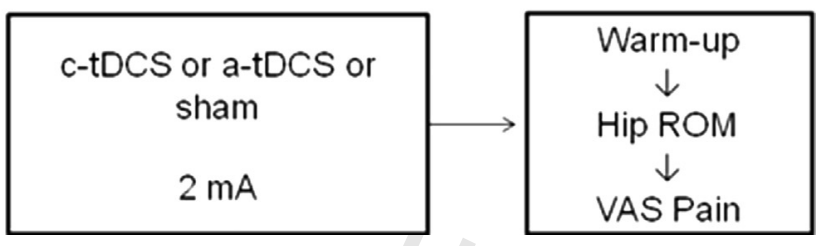

Figure 1 Experimental design.

\subsection{Statistical analyses}

A two-way analysis of variance (Anova) with repeated measures with the entrance for condition (c-tDCS, a-tDCS, and sham) and time (baseline, and post-condition) were performed for the hip ROM and VAS pain. The sphericity assumption was tested using the Mauchly's test and the Greenhouse-Geisser correction was used whenever data sphericity was violated. Post-hoc comparisons were performed using the Bonferroni correction. Values were reported with mean and standard deviation. The level of significance was set at $P \leq 0.05$. The analysis was undertaken using SPSS 23,0.

A bivariate Pearson correlation $(r)$ was performed, in order to determine a possible association between hip ROM and VAS pain measures for the c-tDCS and a-tDCS conditions. This association was determined between post-conditionminus baseline scores in both measures.

Effect size analysis was conducted to report the magnitude of differences between the c-tDCS and a-tDCS conditions compared to sham for hip ROM and VAS pain. Effect sizes were computed using the equation proposed by Morris and De Shon [27], on the G*Power software (version 3.1). Effect sizes were classified as trivial $(d<0.19)$, small $(d=0.20-0.49)$, moderate $(d=0.50-0.79)$, large $(d=0.80-1.29)$, and very large $(d>1.30)$ [28]. In each condition, a descriptive analysis was performed for responders vs. non-responders. To be considered responder, each participant need to simply respond to the training protocol used in the study, and non-responder is the participant that simply do not respond to the training but that doesn't necessarily mean they would not respond to any training program [29]. We use changes from of the baseline to post-condition in each subject for the hip ROM and VAS pain. The absolute values were expressed for each subject.

\section{Results}

Two-way repeated measures Anova's showed significant interactions between condition and time $(F(2,14)=140.36$; $P<0.001)$, and main effect for condition $(F(2,14)=60.17$; $P<0.001)$ in hip ROM. There was no significant main effect for time $(F(1,7)=3.01 ; P>0.05)$. There were no significant differences between the conditions in the baseline (c-tDCS:
$109.0 \pm 4.3^{\circ}$; a-tDCS: $109.8 \pm 4.7^{\circ}$; sham-tDCS: $110.1 \pm 4.6^{\circ}$, $P>0.05)$. In post-condition, c-tDCS $\left(123.9 \pm 4.1^{\circ}\right)$ was greater than a-tDCS $\left(100.4 \pm 1.2^{\circ}, P<0.001\right)$, and shamtDCS $\left(110.7 \pm 4.2^{\circ}, P<0.001\right)$. At this time, sham-tDCS was greater than a-tDCS $(P<0.001)$. There was baseline to post-condition changes in both c-tDCS and a-tDCS conditions. The hip ROM increased in the post-condition compared to baseline in the c-tDCS condition (baseline: $109.0 \pm 4.3^{\circ}$, post-condition: $\left.123.9 \pm 4.1^{\circ}, P<0.001\right)$. In the a-tDCS condition, the hip ROM decreased in the postcondition compared to baseline (baseline: 109.8 $\pm 4.7^{\circ}$, post-condition: $100.4 \pm 1.2^{\circ}, P<0.001$ ) (Fig. 2).

For VAS pain, two-way repeated measures Anova's showed significant interactions between condition and time $(F(2,14)=57.49 ; \quad P<0.001)$, main effect for condition $(F(2,14)=94.25 ; P<0.001)$, and main effect for time $(F(1,7)=56.70 ; P<0.001)$. There were no significant differences between the conditions in the baseline (c-tDCS: $9.5 \pm 0.7$; a-tDCS: $9.6 \pm 0.5$; sham-tDCS: $9.6 \pm 0.5, P>0.05$ ) for pain perception. In post-condition, the pain perception in the c-tDCS $(5.1 \pm 0.8)$ was less than a-tDCS $(9.5 \pm 0.5$, $P<0.001)$, and sham-tDCS $(9.6 \pm 0.5, P<0.001)$. In the $c^{-}$ tDCS condition, the VAS pain decreased in the post-condition compared to baseline (baseline: $9.5 \pm 0.7$, post-condition: $5.1 \pm 0.8^{\circ}, P<0.001$ ) (Fig. 3).

No correlations was showed between post-conditionminus baseline scores in the hip ROM and VAS pain measures for the c-tDCS $(r=0.02, P>0.05)$ and a-tDCS $(r=-0.12$, $P>0.05$ ) conditions.

Means and standard deviation values are shown in Table 1. Effect size was very large in the c-tDCS condition compared to sham-tDCS $(d=2.38)$ and trivial in the a-tDCS condition compared to sham-tDCS $(d=0.07)$ in hip ROM. For VAS pain, effect size was very large in the c-tDCS condition compared to sham-tDCS $(d=-5.05)$ and trivial in the a-tDCS condition compared to sham-tDCS $(\mathrm{d}=0.16)$ (Table 1$)$.

Regarding responders and non-responders was showed that the c-tDCS condition provided an increase in the hip ROM in all subjects (range: 7.9 to $23.5^{\circ}$ ). The a-tDCS condition provided a decrease in hip ROM in all subjects (range: -3.2 to $\left.-17.5^{\circ}\right)$. In the sham-tDCS condition occurred a small increase (range: 1 to $3^{\circ}$ ) and decrease (range: -1.7 to $-2^{\circ}$ ) in hip ROM (Fig. 4).

Responders and non-responders were showed that the ctDCS condition provided a decrease in VAS pain in all subjects pain in sedentary male individuals. Sci sports (2019), https://doi.org/10.1016/j.scispo.2019.01.005 


\section{Hip flexion}

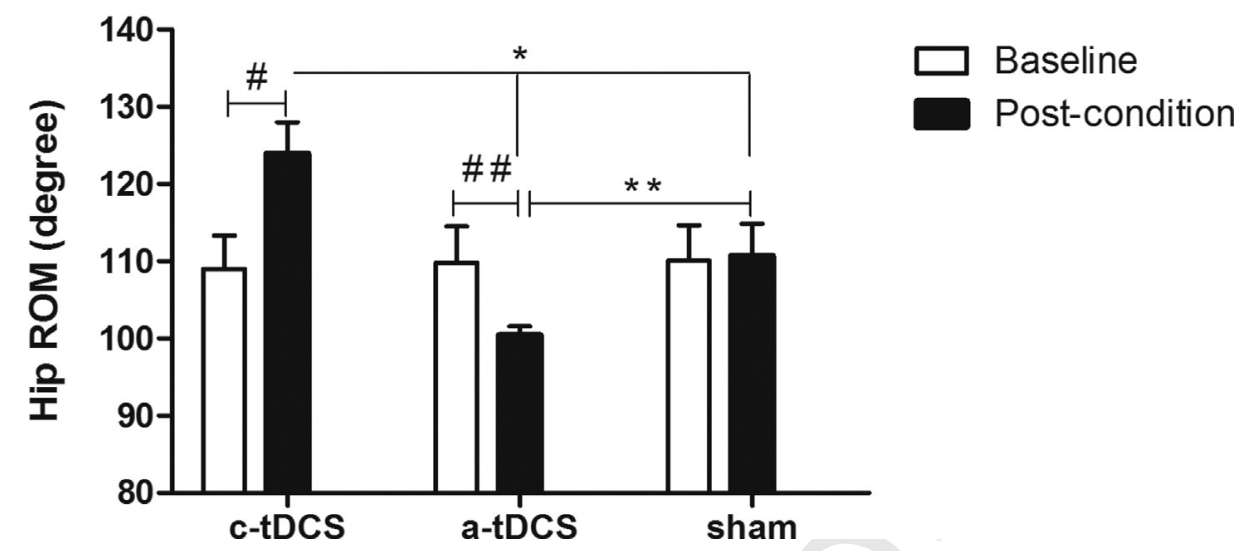

Figure 2 Effects of tDCS on Hip ROM. " $\mathrm{C}$-tDCS $>\mathrm{a}$-tDCS $(P<0.001)$, and sham $(P \leq 0.001)$; ${ }^{* *}$ sham $>\mathrm{a}$-tDCS $(P \leq 0.001)$; ${ }^{\#} \mathrm{C}-\mathrm{tDCS}$ in the post-condition $>\mathrm{C}$-tDCS in the baseline $(P<0.001)$; \# a-tDCS in the baseline $>$ a-tDCS in the post-condition $(P \leq 0.001)$.

\section{Pain perception}

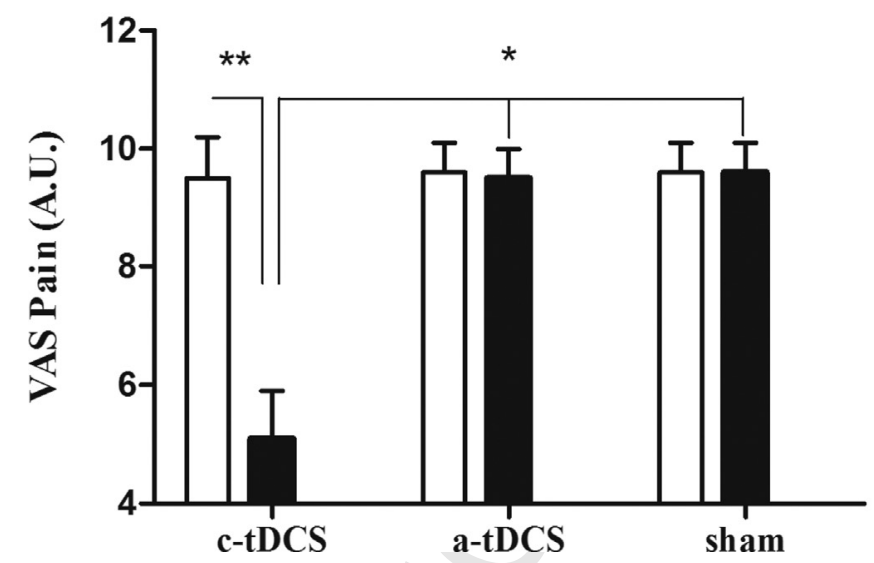

$\square$ Baseline

Post-condition

tDCS in the baseline $(P<0.001)$.

Table 1 Descriptive statistics and effect sizes for ROM of the right hip, and pain perception.

\begin{tabular}{lccc}
\hline Measures & Baseline $(M \pm S D)$ & Post-condition $(M \pm S D)$ & ES vs. sham(classification) \\
\hline $\begin{array}{l}\text { ROM (degrees) } \\
\text { C-tDCS }\end{array}$ & $109.0 \pm 4.3$ & $123.9 \pm 4.1$ & 2.38 (very large) \\
a-tDCS & $109.8 \pm 4.7$ & $100.4 \pm 1.2$ & 0.07 (trivial) \\
sham & $110.1 \pm 4.6$ & $110.7 \pm 4.2$ & \\
VAS Pain (mm) & & & -5.05 (very large) \\
C-tDCS & $9.5 \pm 0.7$ & $5.1 \pm 0.8$ & 0.16 (trivial) \\
a-tDCS & $9.6 \pm 0.5$ & $9.5 \pm 0.5$ & \\
sham & $9.6 \pm 0.5$ & $9.6 \pm 0.5$ & \\
\hline
\end{tabular}

A.U.: Arbitrary Unit. 


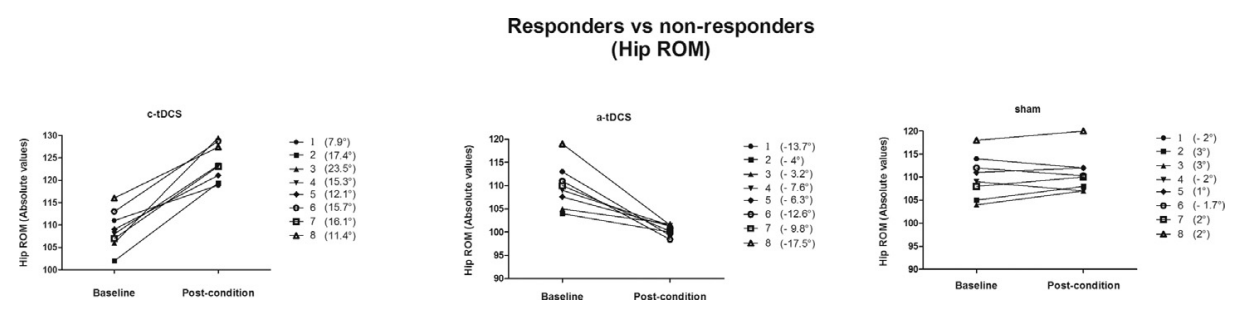

Figure 4 Responders vs. non-responders for Hip ROM.

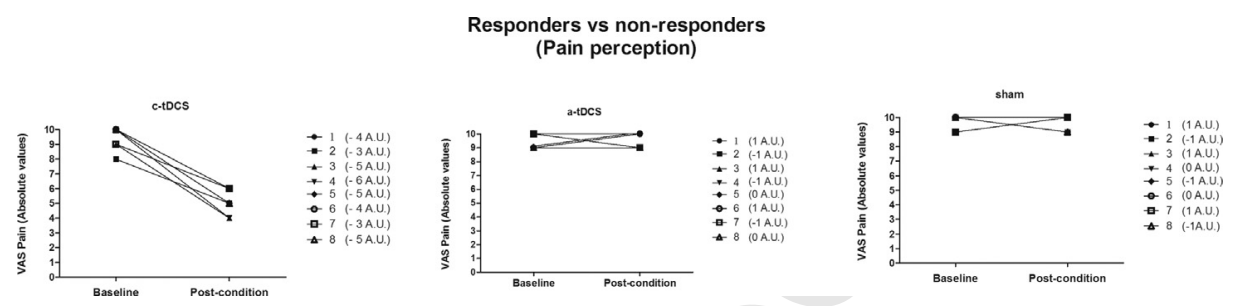

Figure 5 Responders vs. non-responders for pain perception. A.U.: Arbitrary Unit.

(range: -3 to -6 A.U.). The a-tDCS condition showed an increase in two subjects ( 3 , and $6 ; 1$ A.U. for each one), a decrease in three subjects $(2,4$, and $7 ;-1$ A.U. for each one), and three remained unaltered (subjects 1, 5, and 8) for VAS pain. For sham-tDCS condition, occurred a small increase in three subjects (1,3, and 7; 1 A.U. for each one), a decrease in three subjects $(2,5$, and $8 ;-1$ A.U. for each one), and two remained unaltered (subjects 4 and 6 ) for VAS pain (Fig. 5).

\section{Discussion}

The aim of this study was to examine whether the effects of tDCS enhance hip ROM, and pain perception in comparison to a-tDCS and sham-tDCS in a sedentary male. According to our initial hypothesis, results showed that c-tDCS condition improved hip ROM, and decreased VAS pain in comparison to a-tDCS and sham-tDCS. Our findings showed that c-tDCS over the SM1 bilaterally significantly increased hip ROM and reduced VAS pain, while a-tDCS over SM1 bilaterally led to a decrease in hip ROM, and sham-tDCS had no effect.

Just one study analyzed the effects of c-tDCS on flexibility. Mizuno et al. [13] showed that $2 \mathrm{~mA}$ c-tDCS applied to SM1 10 minutes, significantly increased ankle ROM, whereas a-tDCS and sham-tDCS had no effect. Our results were similar by Mizuno et al. [13], despite the different joint assessed. Corroborating our findings, all subjects demonstrated an increase in ROM following c-tDCS (our results, range: 7.1-22.1\%; Mizuno et al.'s results [13], range: $1.0-26.2 \%)$.

After c-tDCS application, hip ROM was likely because of decreased pain perception, considering that the individuals do not stopped hip flexion at the same level of perceived discomfort during the pre- and post-tDCS tests. However, we did not find a positive association between the increase in hip ROM and a reduction in pain perception. A possible explanation is that changes in pain perception could cause a decrease in SM1 excitability after c-tDCS. Many fMRI studies have substantiated the involvement of SM1 in pain perception [30-34]. Peyron et al. [32] revealed that significant SM1 activation after painful stimulation and specific nociceptive neurons are known to exist in SM1 [35]. Furthermore, Antal et al. [36] showed that c-tDCS over the SM1 decreased laser-stimulated subjective pain perception of the hand, whereas anodal and sham-tDCS had no effect. These findings suggest that c-tDCS over the SM1 decreased subjective pain perception. Previous results also indicate that the increase in hip ROM observed in our study may be based on decreased pain perception secondary to decreased brain excitability, which was caused by c-tDCS over the SM1 [19].

In addition, pain perception decreased in all subjects. In fact, SM1 seems to be involved with the perception of pain, according to some studies [37]. Our findings are similar to several studies that applied c-tDCS to SM1 in healthy adults, observing a decrease in pain thresholds [20,33-36,38,39]. fMRI studies have shown that experience of pain coincides with hyperactivity of SM1 $[33-36,38,39]$. The rationale for the reduction of pain by C-tDCS over SM1 is corroborated by some studies $[40,41]$. c-tDCS when applied to $M 1$ and S1 in healthy individuals [42-45], decreases brain excitability $[46,47]$ and increases pain threshold perception in healthy individuals $[44,45]$.

However, new evidence suggests that inhibitory effects of c-tDCS may shift to excitatory effects by modulation of c-tDCS parameters such as current intensity and duration [48], site of stimulation [49], and repetition [50]. This same argument could be used for a-tDCS and could explain our result, i.e., no increase in pain perception. Thus, tDCS intensity does not essentially increase efficacy of stimulation, however, may also modify the direction of excitability alterations [48-50]. This should be taken into account for applications of tDCS using different intensities and durations in order to achieve stronger or longer lasting after-effects.

Some methodological limitations were presented in the study. First, the number of subjects was small, although the effect size calculation was performed. Second, it is possible that the site of the stimulated area has been large, due to 


\section{${ }_{397} Q 7$ Uncited reference}

\section{Conclusion} and readily available.

\section{$[51]$}

\section{Acknowledgments} in English revision.

\section{References} 2018;28:794-806. the size of the electrode $\left(35 \mathrm{~cm}^{2}\right)$. Third, the flexion of the hip was performed with knee extended, limiting our interpretation on biarticular muscles involved in this movement.

This study suggests that c-tDCS applied to SM1 may promote increased in ROM of the hip and decreased and perception of pain. tDCS has been stepping out of the laboratory into the community at large, including the sports and fitness fields. Although its effects are variable between individuals and within individuals, it is not unreasonable to claim that tDCS has great potential as "ergogenic resource" for improving human physical performance, e.g. flexibility and pain perception. In an increasingly success-oriented society with less effort and improved performance, tDCS seems to be a useful tool for use in sports and fitness, as well as safe with regard to its tolerance and adverse effects, relatively inexpensive

\section{Disclosure of interest}

The authors declare that they have no competing interest.

Sergio Machado was supported by grant from Carlos Chagas Foundation for the Research Support in the State of Rio de Janeiro (FAPERJ), Young Scientists from the State of Rio de Janeiro 2017. We thank Dr. Eric Murillo-Rodriguez for his help

[1] Apostolopoulos N, Metsios GS, Flouris AD, Koutedakis Y, Wyon $M A$. The relevance of stretch intensity and position - a systematic review. Front Psychol 2015;6:1128-53.

[2] McHugh MP, Cosgrave $\mathrm{CH}$. To stretch or not to stretch: the role of stretching in injury prevention and performance. Scand J Med Sci Sports 2010;20:169-81.

[3] Rubini EC, Costa AL, Gomes PS. The effects of stretching on strength performance. Sports Med 2007;37:213-24.

[4] Herbert RD, de Noronha M, Kamper SJ. Stretching to prevent or reduce muscle soreness after exercise. Cochrane Database Syst Rev 2011;6 [CD004577].

[5] Freitas SR, Mendes B, Le Sant G, Andrade RJ, Nordez A, Milanovic Z. Can chronic stretching change the muscle-tendon mechanical properties? A review. Scand J Med Sci Sports

[6] Guissard N, Duchateau J. Neural aspects of muscle stretching. Exerc Sport Sci Rev 2006;34:154-8.

[7] Trajano GS, Seitz LB, Nosaka K, Blazevich AJ. Can passive stretch inhibit motoneuron facilitation in the human plantar flexors? J Appl Physiol 2014;117:1486-92.

[8] Guissard N, Duchateau J. Effect of static stretch training on neural and mechanical properties of the human plantar-flexor muscles. Muscle Nerve 2004;29:248-55.
[9] Weppler $\mathrm{CH}$, Magnusson SP. Increasing muscle extensibility: a matter of increasing length or modifying sensation? Phys Ther 2010;90:438-49.

[10] Medeiros DM, Cini A, Sbruzzi G, Lima CS. Influence of static stretching on hamstring flexibility in healthy young adults: systematic review and meta-analysis. Physiother Theory Pract 2016;32:438-45.

[11] Medeiros DM, Martini TF. Chronic effect of different types of stretching on ankle dorsiflexion range of motion: Systematic review and meta-analysis. Foot 2017;34:28-35.

[12] Mazor M, Lee JQ, Peled A, Zerzan S, Irwin C, Chesney MA, et al. The effect of yoga on arm volume, strength, and range of motion in women at risk for breast cancer-related lymphedema. J Altern Complement Med 2017;24:154-60.

[13] Mizuno T, Aramaki Y. Cathodal transcranial direct current stimulation over the $\mathrm{Cz}$ increases joint flexibility. Neurosci Res 2017;114:55-61.

[14] Nitsche MA, Liebetanz D, Tergau F, Paulus W. Modulation of cortical excitability by transcranial direct current stimulation. Der Nervenarzt 2002;73:332-5.

[15] Nitsche MA, Paulus W. Excitability changes induced in the human motor cortex by weak transcranial direct current stimulation. J Physiol 2000;527:633-9.

[16] Lattari E, Andrade ML, Filho AS, Moura AM, Neto GM, Silva JG, et al. Can transcranial direct current stimulation improves the resistance strength and decreases the rating perceived scale in recreational weight-training experience? J Strength Cond Res 2016;30:3381-7.

[17] Lattari E, Campos C, Lamego MK, Passos de Souza SL, Neto GM, Rocha NB, et al. Can transcranial direct current stimulation improve muscle power in individuals with advanced resistance training experience? J Strength Cond Res 2017.

[18] Lattari E, de Oliveira BS, Oliveira BRR, de Mello Pedreiro RC, Machado S, Neto GAM. Effects of transcranial direct current stimulation on time limit and ratings of perceived exertion in physically active women. Neurosci Lett 2018;662:12-6.

[19] Vaseghi B, Zoghi M, Jaberzadeh S. Does anodal transcranial direct current stimulation modulate sensory perception and pain? A meta-analysis study. Clin Neurophysiol 2014;125:1847-58.

[20] Vaseghi B, Zoghi M, Jaberzadeh S. Differential effects of cathodal transcranial direct current stimulation of prefrontal, motor and somatosensory cortices on cortical excitability and pain perception - a double-blind randomised sham-controlled study. Eur J Neurosci 2015;42:2426-37.

[21] American College of Sports Medicine. ACSM's guidelines for exercise testing and prescription. Philadelphia: Wolters Kluwer/Lippincott Williams \& Wilkins; 2013.

[22] Jasper H. Report of committee on methods of clinical examination in eletroencephalography. Eletroencephalogr Clin Neurophysiol 1958;10:370-5.

[23] Gandiga PC, Hummel FC, Cohen LG. Transcranial DC stimulation (tDCS): a tool for double-blind sham-controlled clinical studies in brain stimulation. Clin Neurophysiol 2006;117: 845-50.

[24] Boggio PS, Zaghi S, Lopes M, Fregni F. Modulatory effects of anodal transcranial direct current stimulation on perception and pain thresholds in healthy volunteers. Eur $\mathrm{J}$ Neurol 2008;15:1124-30.

[25] Williamson A, Hoggart B. Pain: a review of three commonly used pain rating scales. J Clin Nurs 2005;14:798-804.

[26] Jensen MP, Karoly P, Braver S. The measurement of clinical pain intensity: a comparison of six methods. Pain 1986;27:117-26.

[27] Morris SB, DeShon RP. Combining effect size estimates in metaanalysis with repeated measures and independent-groups designs. Psychol Methods 2002;7:105-25.

[28] Rosenthal JA. Qualitative descriptors of strength of association and effect size. J Soc Serv Res 1996;21:37-59. 
[29] Pickering C, Kiely J. Do non-responders to exercise exist-and if so, what should we do about them? Sports Med 2018.

[30] Bingel U, Lorenz J, Glauche V, Knab R, Glascher J, Weiller $C$, et al. Somatotopic organization of human somatosensory cortices for pain: a single Trial fMRI study. Neuroimage 2004;23:224-32.

[31] Bushnell MC, Duncan GH, Hofbauer RK, Ha B, Chen JI, Carrier B. Pain perception: is there a role for primary somatosensory cortex? Proc Natl Acad Sci U S A 1999;96:7705-9.

[32] Forss N, Raij TT, Seppa M, Hari R. Common cortical network for first and second pain. Neuroimage 2005;24:132-42.

[33] Peyron R, Laurent B, Garcia-Larrea L. Functional imaging of brain responses to pain. A review and meta-analysis (2000). Neurophysiol Clin 2000;30:263-88.

[34] Porro CA, Baraldi P, Pagnoni G, Serafini M, Facchin P, Maieron $M$, et al. Does anticipation of pain affect cortical nociceptive systems? J Neurosci 2002;22:3206-14.

[35] Kenshalo Jr DR, Isensee O. Responses of primate SI cortical neurons to noxious stimuli. J Neurophysiol 1983;50:1479-96.

[36] Antal A, Brepohl N, Poreisz C, Boros K, Csifcsak G, Paulus W. Transcranial direct current stimulation over somatosensory cortex decreases experimentally induced acute pain perception. Clin J Pain 2008;24:56-63.

[37] Magnusson SP. Passive properties of human skeletal muscle during stretch maneuvers. A review. Scand J Med Sci Sports 1998;8:65-77.

[38] Bornhovd K, Quante M, Glauche V, Bromm B, Weiller C, Buchel C. Painful stimuli evoke different stimulus-response functions in the amygdala, prefrontal, insula and somatosensory cortex: a single-trial fMRI study. Brain 2002;125:1326-36.

[39] Apkarian AV, Bushnell MC, Treede RD, Zubieta JK. Human brain mechanisms of pain perception and regulation in health and disease. Eur J Pain-London 2005;9:463-84.

[40] Koyama T, McHaffie JG, Laurienti PJ, Coghill RC. The subjective experience of pain: where expectations become reality. Proc Natl Acad Sci USA 2005;102:12950-5.

[41] Anderson WS, O'Hara S, Lawson HC, Treede RD, Lenz FA. Plasticity of pain-related neuronal activity in the human thalamus. Prog Brain Res 2006;157:353-64.
[42] Terney D, Bergmann I, Poreisz C, Chaieb L, Boros K, Nitsche $M A$, et al. Pergolide increases the efficacy of cathodal direct current stimulation to reduce the amplitude of laser-evoked potentials in humans. J Pain Symptom Manag 2008;36:79-91.

[43] Csifcsak G, Antal A, Hillers F, Levold M, Bachmann CG, Happe $\mathrm{S}$, et al. Modulatory effects of transcranial direct current stimulation on laser-evoked potentials. Pain Med 2009;10:122-32.

[44] Bachmann CG, Muschinsky S, Nitsche MA, Rolke R, Magerl W, Treede RD, et al. Transcranial direct current stimulation of the motor cortex induces distinct changes in thermal and mechanical sensory percepts. Clin Neurophysiol 2010;121:2083-9.

[45] Grundmann L, Rolke R, Nitsche MA, Pavlakovic G, Happe $S$, Treede RD, et al. Effects of transcranial direct current stimulation of the primary sensory cortex on somatosensory perception. Brain Stimul 2011;4:253-60.

[46] Nitsche M, Paulus W. Excitability changes induced in the human motor cortex by weak transcranial direct current stimulation. J Physiol London 2000;527:633-9.

[47] Matsunaga K, Nitsche MA, Tsuji S, Rothwell JC. Effect of transcranial DC sensorimotor cortex stimulation on somatosensory evoked potentials in humans. Clin Neurophysio 2004;115:456-60.

[48] Batsikadze G, Moliadze V, Paulus W, Kuo MF, Nitsche MA. Partially non-linear stimulation intensity-dependent effects of direct current stimulation on motor cortex excitability in humans. J Physiol London 2013;591:1987-2000.

[49] Boros K, Poreisz C, Munchau A, Paulus W, Nitsche MA. Premotor transcranial direct current stimulation (tDCS) affects primary motor excitability in humans. Eur J Neurosci 2008;27:1292-300.

[50] Monte-Silva K, Kuo MF, Liebetanz D, Paulus W, Nitsche MA. Shaping the optimal repetition interval for cathodal transcranial direct current stimulation (tDCS). J Neurophysiol 2010;103:1735-40.

[51] Marfell-Jones M, Olds T, Stewart A, Carter L. International standards for anthropometric assessment. Palmerston North: ISAK; 2006. 